\title{
Quantum confined peptide assemblies with tunable visible to near-infrared spectral range
}

\author{
Kai Tao (1) ', Zhen Fan 2,3,4, Leming Sun ${ }^{5}$, Pandeeswar Makam¹, Zhen Tian, Mark Ruegsegger², \\ Shira Shaham-Niv', Derek Hansford², Ruth Aizen', Zui Pan (10 7, Scott Galster ${ }^{8}$, Jianjie Ma ${ }^{3,9}$, Fan Yuan ${ }^{10}$, \\ Mingsu Si ${ }^{11}$, Songnan $\mathrm{Qu}^{6}$, Mingjun Zhang ${ }^{2,3,12}$, Ehud Gazit,13 \& Junbai Li (iD ${ }^{14}$
}

Quantum confined materials have been extensively studied for photoluminescent applications. Due to intrinsic limitations of low biocompatibility and challenging modulation, the utilization of conventional inorganic quantum confined photoluminescent materials in bioimaging and bio-machine interface faces critical restrictions. Here, we present aromatic cyclo-dipeptides that dimerize into quantum dots, which serve as building blocks to further self-assemble into quantum confined supramolecular structures with diverse morphologies and photoluminescence properties. Especially, the emission can be tuned from the visible region to the near-infrared region ( $420 \mathrm{~nm}$ to $820 \mathrm{~nm}$ ) by modulating the self-assembly process. Moreover, no obvious cytotoxic effect is observed for these nanostructures, and their utilization for in vivo imaging and as phosphors for light-emitting diodes is demonstrated. The data reveal that the morphologies and optical properties of the aromatic cyclodipeptide self-assemblies can be tuned, making them potential candidates for supramolecular quantum confined materials providing biocompatible alternatives for broad biomedical and opto-electric applications.

\footnotetext{
${ }^{1}$ Department of Molecular Microbiology and Biotechnology, George S. Wise Faculty of Life Sciences, Tel Aviv University, 6997801 Tel Aviv, Israel.

${ }^{2}$ Department of Biomedical Engineering, College of Engineering, The Ohio State University, Columbus, OH 43210, USA. ${ }^{3}$ Dorothy M. Davis Heart and Lung Research Institute, The Ohio State University Wexner Medical Center, Columbus, OH 43210, USA. ${ }^{4}$ Department of Polymeric Materials, School of Materials Science and Engineering, Tongji University, 201804 Shanghai, China. ${ }^{5}$ School of Life Sciences, Northwestern Polytechnical University, 710065 Xi'an, China. ${ }^{6}$ State Key Laboratory of Luminescence and Applications, Changchun Institute of Optics, Fine Mechanics and Physics, Chinese Academy of Sciences, 130033 Changchun, China. ${ }^{7}$ College of Nursing and Health Innovation, University of Texas at Arlington, Arlington, TX 76019, USA. ${ }^{8}$ Rockefeller Neuroscience Institute and Department of Neuroscience, WVU School of Medicine, 9100 Morgantown, West Virginia, USA. ${ }^{9}$ Department of Surgery, The Ohio State University, Columbus 43210, USA. ${ }^{10}$ Department of Biomedical Engineering, Duke University, Durham, NC 27708, USA. ${ }^{11}$ Key Laboratory for Magnetism and Magnetic Materials of MOE, Key Laboratory of Special Function Materials and Structure Design, Ministry of Education, Lanzhou University, 730000 Lanzhou, China. ${ }^{12}$ Nuroulogical Institute and Department of Surgery, The Ohio State University, Columbus, OH 43210, USA. ${ }^{13}$ Department of Materials Science and Engineering, Iby and Aladar Fleischman Faculty of Engineering, Tel Aviv University, 6997801 Tel Aviv, Israel. ${ }^{14}$ Beijing National Laboratory for Molecular Sciences, CAS Key Lab of Colloid, Interface and Chemical Thermodynamics, Institute of Chemistry, Chinese Academy of Sciences, 100190 Beijing, China. These authors contributed equally: Kai Tao, Zhen Fan Correspondence and requests for materials should be addressed to

M.Z. (email: zhang.4882@osu.edu) or to E.G. (email: ehudg@post.tau.ac.il) or to J.L. (email: jbli@iccas.ac.cn)
} 
O uantum confined (QC) materials, such as quantum dots (QDs), have been widely employed for imaging due to their remarkable photoluminescent properties ${ }^{1-4}$. However, the state-of-the-art inorganic QC constituents, such as cadmium-based QDs, are intrinsically cytotoxic, thus extremely limiting their applications $s^{5,6}$. Although organic fluorescent dyes allow to overcome the potential cytotoxicity to some extent, several concerns, including weak sustainability and photobleaching, narrow color spectrum, and in some cases complicated synthesis procedures, still practically impede their utilization ${ }^{4,7}$. The quest for eco-friendly, organic, tunable, and flexible QC alternatives with improved and stable photoluminescence is continuously ongoing ${ }^{8-10}$.

Accumulating studies demonstrate that aromatic lineardipeptides, with the representative model of diphenylalanine $(\mathrm{FF})^{11}$, can self-assemble into nanostructures with remarkable physiochemical features $^{12,13}$, such as optic ${ }^{14,15}$, electrical ${ }^{16}$, ferroelectric $^{17}$ (including piezoelectric ${ }^{18}$ and pyroelectric ${ }^{19}$ ) properties $^{20-23}$. Especially, the supramolecular morphologies and properties can be easily modified by amino acids substitutions 24 , covalent conjugation $^{25}$ or co-assembly with external moieties ${ }^{26,27}$. For example, upon substitution of one $\mathrm{F}$ with tryptophan (W), self-assembling FW nanostructures present a smaller bandgap of $2.25 \mathrm{eV}$, compared to $3.25 \mathrm{eV}$ of $\mathrm{FF}$ nanotubes ${ }^{28}$, thus showing improved conductive ${ }^{29}$ and photoluminescent properties ${ }^{24}$. Moreover, recent studies revealed that cyclo-dipeptides with backbones of 2,5-diketopiperazine configurations, derived from dehydration condensation of linear dipeptides ${ }^{16,30}$, self-assemble into photoluminescent nanostructures different from their linear counterparts $^{31,32}$.

Here, we demonstrate the modulation of $\mathrm{W}$-based aromatic cyclo-dipeptides self-assemblies into QC microstructures with photoluminescent properties. In particular, the emissions are tuned throughout the visible and near-infrared region by modifying the self-assembly process. These wide spectral range QC structures can thus be used as bio-inspired phosphors for the fabrication of light-emitting diodes (LEDs) and for in vivo bioimaging without any obvious cytotoxicity. The results reveal that the morphologies and optical properties of the $\mathrm{W}$-based aromatic cyclo-dipeptide self-assemblies can be tuned to achieve broad fluorescence emissions, making them promising candidates as supramolecular QC materials for biomedical and opto-electric applications.

\section{Results}

Dimeric QDs as building blocks for self-assembly. Aiming to examine their potential as QC materials, we characterized the photoluminescent properties of two tryptophan (W)-containing aromatic cyclo-dipeptides ${ }^{33}$, cyclo-phenylalanine-tryptophan (cyclo-FW), and cyclo-WW (Fig. 1a), both dissolved in methanol $(\mathrm{MeOH})$. Fluorescent characterization demonstrated red shifts of the molecular excitation to $305 \mathrm{~nm}$, compared to $285 \mathrm{~nm}$ for the monomers (Fig. 1b), indicating that the cyclo-dipeptides indeed self-assembled, which was further confirmed by dynamic light scattering (DLS) detections (Supplementary Fig. 1). UV-Vis absorption spectra revealed that the cyclo-dipeptides had spikelike absorbance, showing three peaks at $273 \mathrm{~nm}, 280 \mathrm{~nm}$, and $289 \mathrm{~nm}$ (Fig. 1c), characteristic of the formation of QD structures $^{34,35}$. The diameter of the QDs was calculated to be $\sim 2.24 \mathrm{~nm}$ (for calculation details, see Methods), around two-fold of the dimension of the monomer. In addition, mass spectrometry (MS) analysis detected the dimeric molecular weights (MW), along with the corresponding monomeric molecular mass (Fig. 1d), indicating the presence of dimers. Therefore, it can be concluded that the cyclo-dipeptide monomers first formed dimers, which behaved as QDs and served as the fundamental building blocks to further organize into supramolecular structures (Fig. 1e). Theoretical calculations using density functional theory demonstrated that the spatial distributions of the highest occupied and lowest unoccupied molecular orbitals of the dipeptides were mainly concentrated on the side-chain indole rings (Fig. 1f). Specifically, the band gaps $(\Delta E)$ of cyclo-FW and cyclo-WW were calculated to be $3.63 \mathrm{eV}$ and $3.56 \mathrm{eV}$, respectively, indicating their wide-gap semiconductive nature ${ }^{36,37}$. This implies that the dimerization was mostly driven by $\pi-\pi$ interactions between aromatic sidechains, especially the indole rings. Particularly, the aromatic interactions could induce the through-space conjugation of the electron clouds from adjacent indole rings, thus restricting the molecular motions ${ }^{38,39}$ and underlying the molecular basis for QC regions ${ }^{34}$. The QC effects and restricted molecular activities resulted in the release of excitation energy exclusively as emitted light ${ }^{10,12}$

Modulation of the self-assemblies morphology and visible fluorescence. The orderly organized QDs inside the supramolecular self-assemblies result in extensive QC effects along with intrinsic photoluminescent properties ${ }^{12}$. When excited at $370 \mathrm{~nm}$, the cyclo-dipeptides solutions displayed fluorescence in the visible region, with emission at $460 \mathrm{~nm}$ for cyclo-FW and at $425 \mathrm{~nm}$, accompanied by a smaller peak at $520 \mathrm{~nm}$, for cyclo-WW (Fig. 2a). Correspondingly, the solutions showed blue-green color under UV light (365 nm) (Fig. 2a, insets). Scanning electron microscopy (SEM) revealed that the two cyclo-dipeptides selfassembled into distinct supramolecular structures, as needle-like crystals were formed by cyclo-FW (Fig. 3a), while cyclo-WW assembled into spherical nanoparticles (Fig. 3b). The maximal emission of the cyclo-FW assemblies demonstrated a red-shift as excitation wavelength was increased (Supplementary Fig. 2a), indicating that heterogeneous superstructures of different sizes, arising from the dynamic self-organization, co-existed in the solution $^{40,41}$.

The ease of complexation of indole rings suggests that these self-assemblies could be doped by coordination with metal ions ${ }^{42}$. Previous studies revealed that $\mathrm{Zn}(\mathrm{II})$ can participate in amphiphilic peptides self-assembly $24,43,44$ and interferes with amyloid proteins aggregation ${ }^{45,46}$. Indeed, by introducing $\mathrm{Zn}$ (II), the emission of cyclo-WW assemblies (cyclo-WW $+\mathrm{Zn}(\mathrm{II})$ ) was clearly enhanced, showing a narrow peak at $520 \mathrm{~nm}$ with a full width at half maximum of only $18 \mathrm{~nm}$ (Fig. 2b), leading to a luminous green color under UV light and a quantum yield (QY) of $16 \%$ (Fig. 2b, inset). Intriguingly, atomic force microscopy (AFM) experiment indicated the presence of only small nanoparticles, $\sim 3.0 \mathrm{~nm}$ in diameter (Fig. 3c; Supplementary Fig. 3), approximately the dimension of a dimer. Correspondingly, DLS analysis showed that the size of the structures was $\sim 2.88 \mathrm{~nm}$, with no larger particles present (Supplementary Fig. 4). The uniform size distribution resulted in a consistent maximal emission, regardless of excitation wavelength (Supplementary Fig. 2b). These findings demonstrated that following the coordination with $\mathrm{Zn}(\mathrm{II})$, the cyclo-WW self-assembly halted at the dimerization stage.

Another strategy to modulate the supramolecular photoluminescence is to substitute the constituents with their enantiomers ${ }^{47}$. When $\mathrm{W}$ was replaced with its $D$-type enantiomer in cyclo-FW, hereby designated cyclo-F $w$, the fluorescent emission shifted to $430 \mathrm{~nm}$ (Fig. 2c). SEM and DLS analyses revealed that cyclo-F $w$ self-assembled into multi-branched nano-flower architectures (Fig. 3d; Supplementary Figs. 5a, 6a), showing red-shifted maximal emission upon various excitation wavelengths (Supplementary Fig. 2c). 
a

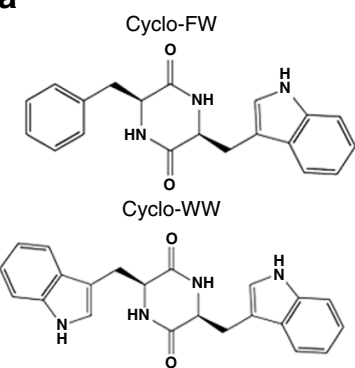

b

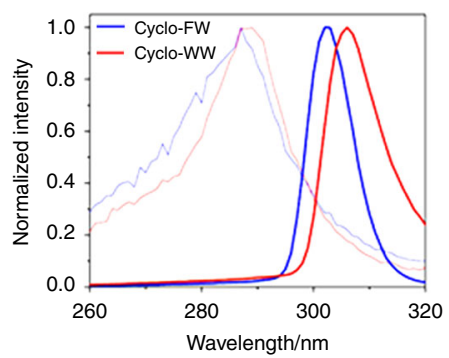

C

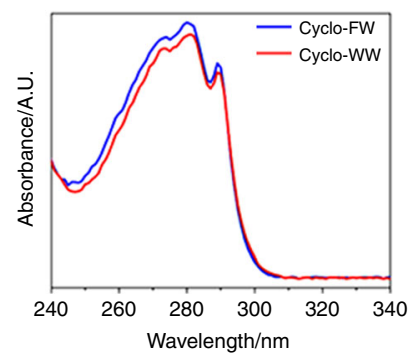

d

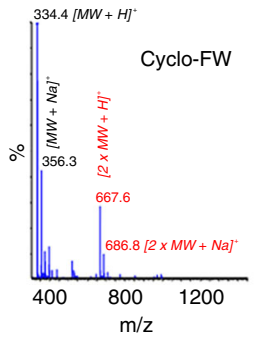

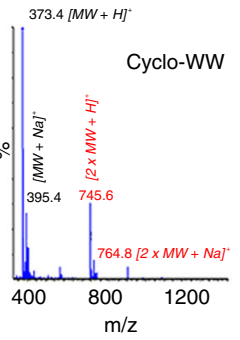

e

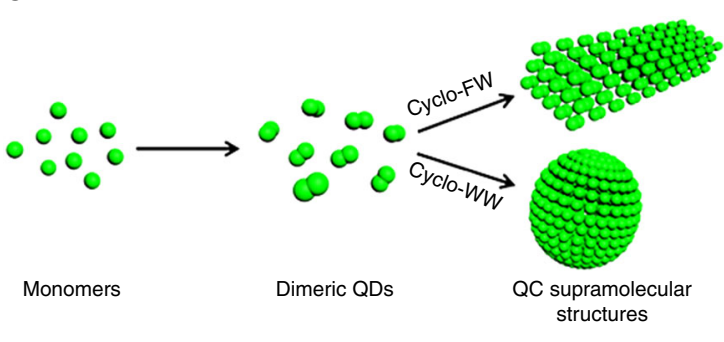

f
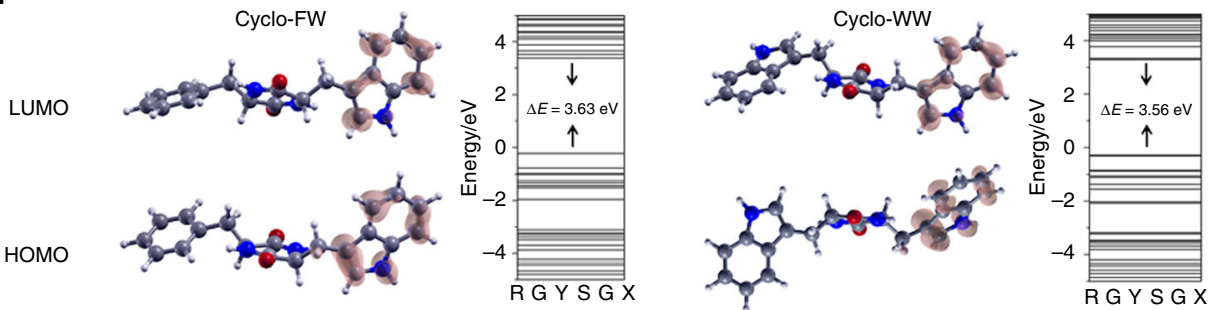

Fig. 1 Cyclo-dipeptide dimers serve as QDs to self-assemble into QC nanostructures. a Molecular structures of cyclo-FW and cyclo-WW. b Excitation spectra of cyclo-dipeptide monomers (thin light curves, $0.05 \mathrm{mM}$ ) and self-assemblies (thick dark curves, $5.0 \mathrm{mM}$ ) in $\mathrm{MeOH}$. The excitation wavelengths red shifted from $285 \mathrm{~nm}$ to $\sim 305 \mathrm{~nm}$ after self-assembly. c UV-Vis absorption spectra of the cyclo-dipeptides after self-assembly, showing spike-like absorptions at $273 \mathrm{~nm}, 280 \mathrm{~nm}$, and $289 \mathrm{~nm}$, characteristic of the formation of QD structures. d MS spectra of cyclo-FW and cyclo-WW at $5.0 \mathrm{mM}$ in $\mathrm{MeOH}$. The dimeric MW is marked in red, and the monomeric MW is marked in black. e Schematic representing the process of cyclo-dipeptides selfassembly: the monomers form dimeric QDs, which serve as the building blocks to self-assemble into larger QC architectures. $\mathbf{f}$ Calculated molecular orbital amplitude plots and energy levels of the highest occupied and lowest unoccupied molecular orbitals of cyclo-FW and cyclo-WW, showing band gaps of $3.63 \mathrm{eV}$ and $3.56 \mathrm{eV}$, respectively

Furthermore, the well-established reductive property of $\mathrm{W}^{48}$ could be utilized to modulate the fluorescence of the cyclodipeptides self-assemblies. When introducing $\mathrm{Cu}(\mathrm{II})$, a weak oxidant $^{49}$, into cyclo-WW assemblies (cyclo-WW $+\mathrm{Cu}(\mathrm{II})$ ), a fluorescent emission appeared at $465 \mathrm{~nm}$, with a QY of $8 \%$ (Fig. 2d). A similar emission was also observed after irradiation with UV light $(365 \mathrm{~nm})$ (cyclo-WW $+\mathrm{UV}$ ) due to the UVinduced radical oxidation (Fig. 2e), implying a potential for photo-stimulated applications. HPLC analysis confirmed that the conversion was complete and the oxidized cyclo-WW was pure (Supplementary Fig. 7). Fluorescent decay experiments demonstrated that after oxidation, the lifetime increased (6.3 ns for cyclo-WW $+\mathrm{Cu}(\mathrm{II}), 8.0 \mathrm{~ns}$ for cyclo-WW $+\mathrm{UV}$ ) compared to cyclo-WW (5.6 ns) (Fig. 2f; Supplementary Fig. 8a), confirming that the redox reactions indeed took place. SEM and DLS characterizations showed that the oxidized cyclo-WW selfassembled into spherical nanoparticles, several hundred nanometers to micrometers in diameter (Fig. 3e, f; Supplementary Figs. 5b, c). In both cases, the maximal emission red-shifted (Supplementary Figs. 2d, e), demonstrating that the spherical nanoparticles further aggregated to form larger particles (Supplementary Figs. 6b, c). In fact, massive precipitates could be found at the bottom of the cyclo-WW $+\mathrm{UV}$ and cyclo-WW $+\mathrm{Cu}$ (II) solutions after one week and one month, respectively.
Mechanisms underlying the fluorescence modulations. Understanding the mechanisms underlying the modulation of the fluorescent properties of the self-assemblies is critical for their future applications ${ }^{50}$. A absorption peak at $515 \mathrm{~nm}$, corresponding to ligand (peptide)-to-metal charge transfer ${ }^{51}$, emerged upon mixing cyclo-WW and $\mathrm{Zn}$ (II) in solution (Fig. 4a), indicating the formation of coordinated architectures. Notably, this absorption peak resulted in a color change from the original white/light yellow of cyclo-WW to pink (Supplementary Fig. 9). In contrast, no new band appeared when $\mathrm{Zn}$ (II) was introduced into a monomeric cyclo-WW solution (Supplementary Fig. 10), confirming that the dimers were indeed the form that complexed with $\mathrm{Zn}(\mathrm{II})$. Especially, the charge transfer could deliver the excited electrons, resulting in reduced fluorescence decay time, from $5.6 \mathrm{~ns}$ of cyclo-WW to $3.6 \mathrm{~ns}$ (Fig. 2f; Supplementary Fig. 8a). To determine the stoichiometric ratio of cyclo-WW and Zn(II), a Job Plot analysis was performed, showing the intersection point at a cyclo-WW proportion of $\sim 0.7$ (Fig. $4 \mathrm{~b}$ ). This result indicates that the stoichiometry of cyclo-WW dimers and $\mathrm{Zn}$ (II) was approximately 1:1 (0.35:0.3). The ${ }^{1} \mathrm{H}-\mathrm{NMR}$ spectra revealed that after coordination, the hydrogen atoms in the backbone showed downfield shifting (Fig. 4c; Supplementary Fig. 11), indicating that the shielding effect became weaker. This suggests that the diketopiperazine ring contributed to the coordination by 
a

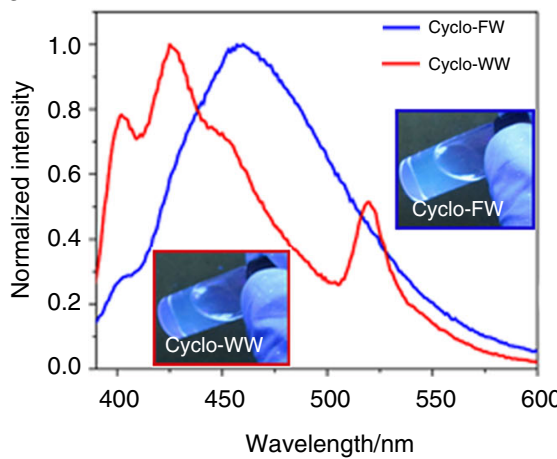

d

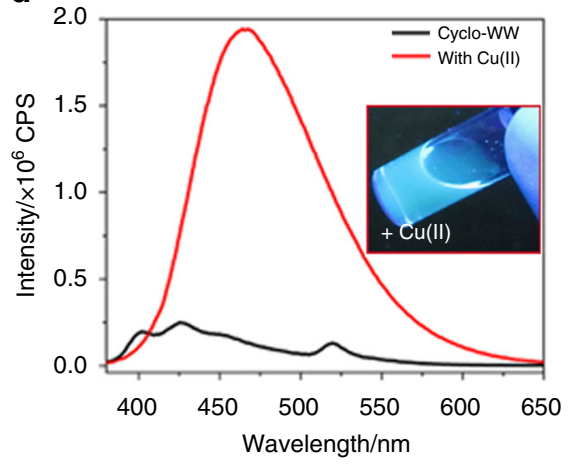

b
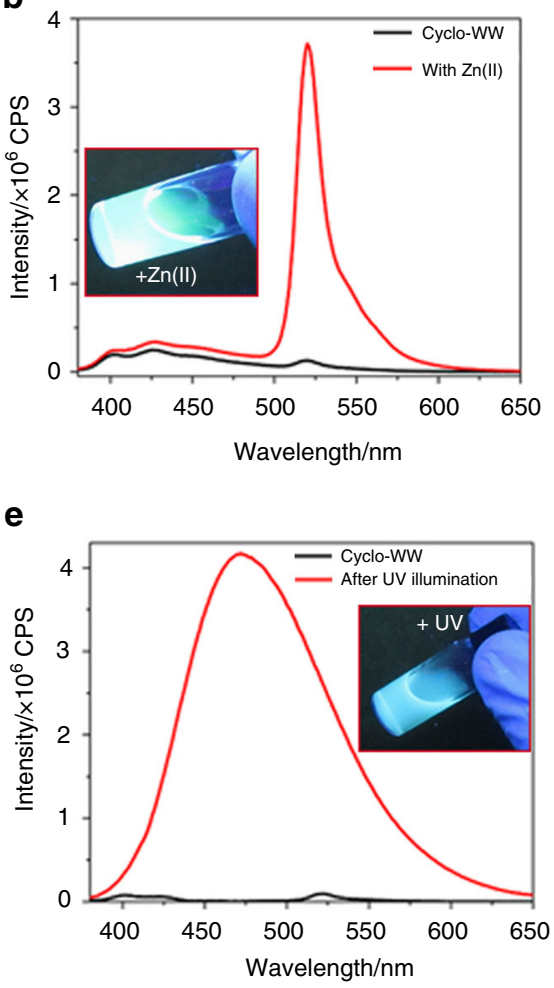

C

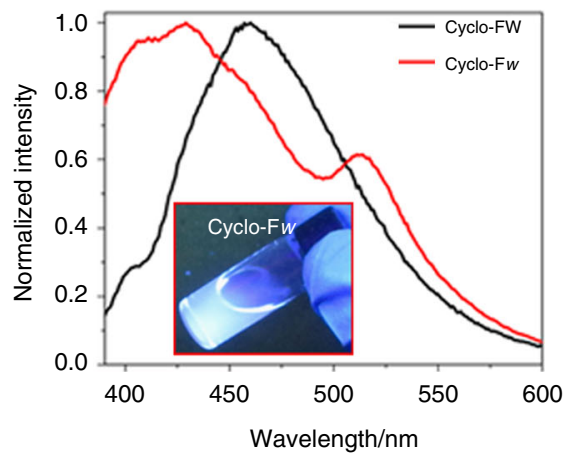

$\mathbf{f}$

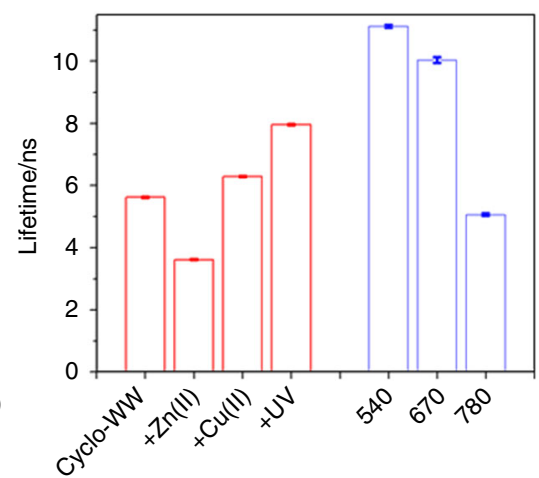

Fig. 2 Visible fluorescence properties and modulation of the self-assemblies. a-e Fluorescent emission of: (a) cyclo-FW (blue) and cyclo-WW (red) selfassemblies, (b) cyclo-WW + Zn(II), (c) cyclo-FW, (d) cyclo-WW + Cu(II), (e) cyclo-WW + UV. The insets show the corresponding solutions under UV light $(365 \mathrm{~nm})$. In b-e, intrinsic fluorescent emissions of the corresponding cyclo-dipeptides were added and marked in black for comparison. $\mathbf{f}$ Lifetime statistics of cyclo-WW self-assemblies. Left panel (red): cyclo-WW in MeOH (cyclo-WW: 370/425; + Zn(II): 370/520; + Cu(II): 370/465; + UV: 370/ 465). Right panel (blue): cyclo-WW + Zn(II) in DMSO (540/610; 670/712; 740/817). Error bars on lifetime measures show standard deviations for three replicates
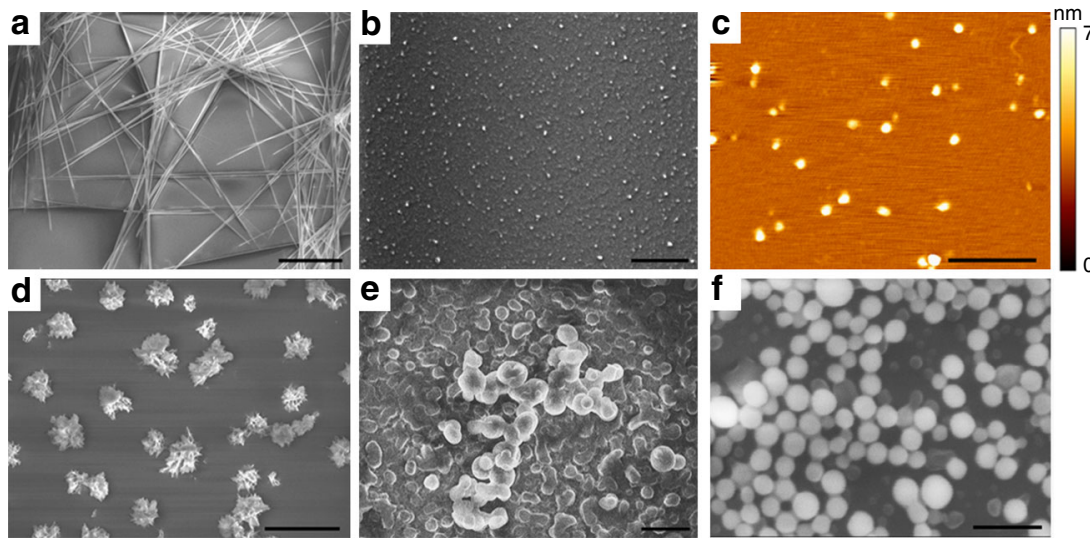

Fig. 3 SEM and AFM images of the cyclo-dipeptides QC self-assemblies in MeOH. a Needle-like cyclo-FW crystals., Scale bar: $40 \mu$ m. b Spherical cycloWW nanoparticles, Scale bar: $20 \mu \mathrm{m}$. c Dimeric QDs of cyclo-WW + Zn(II), Scale bar: $500 \mathrm{~nm}$. d Nano-flower architectures of cyclo-FW, Scale bar: $20 \mu \mathrm{m}$. e and $\mathbf{f}$ Larger spherical nanoparticles of (e) cyclo-WW $+\mathrm{Cu}(\mathrm{II})$ and (f) cyclo-WW + UV. Scale bar: $2 \mu \mathrm{m}$

supplying electrons to interact with $\mathrm{Zn}(\mathrm{II})^{52}$. In contrast, the hydrogen atoms in the indole rings showed upfield shifting (Fig. 4c; Supplementary Fig. 11), indicating that the delocalization of the $\pi$-electrons on the aromatic rings became stronger ${ }^{52}$. This demonstrates that the indole rings formed aromatic interactions with each other and through-space conjugation of electrons took place, consistent with the theoretical calculations. Fouriertransform infrared spectroscopy (FTIR) characterizations demonstrated that in the presence of $\mathrm{Zn}(\mathrm{II})$, the $\mathrm{N}-\mathrm{H}$ stretching vibration in diketopiperazine rings red-shifted from the original $3200 \mathrm{~cm}^{-1}$ to $3071 \mathrm{~cm}^{-1} 53$ due to the decrease of the bond energies resulting from the metal ion adsorption (Fig. 4d, peak 1) 54 , indicating that the nitrogen atoms in the backbone diketopiperazine rings contributed to the complexation with $\mathrm{Zn}$ (II). Combined with the NMR analysis, a plausible molecular mechanism of cyclo-WW coordination with $\mathrm{Zn}$ (II) is depicted in Fig. 4e. The $\mathrm{Zn}(\mathrm{II})$ ion is embedded in a dimer of cyclo-WW, coordinating with two diketopiperazine rings, while the 
a

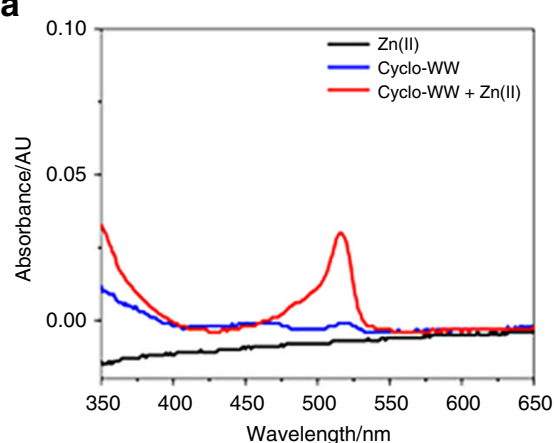

b

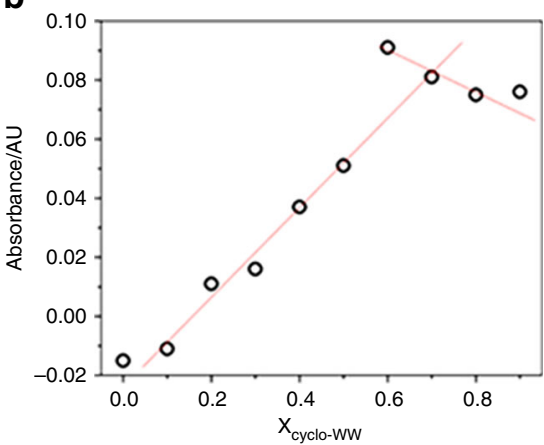

C

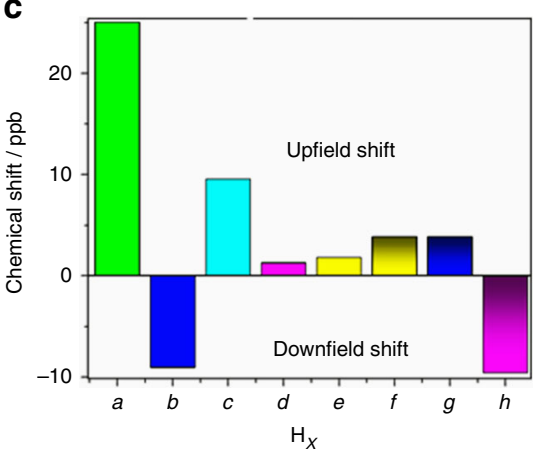

d

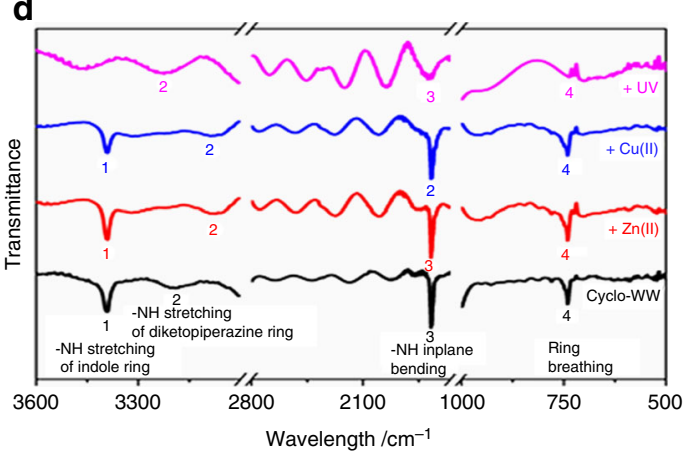

e
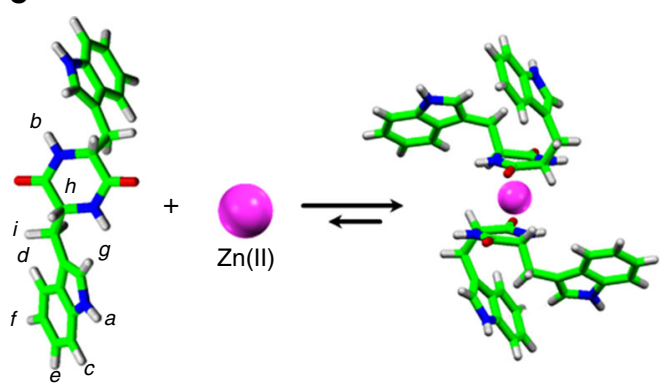

$\mathbf{f}$

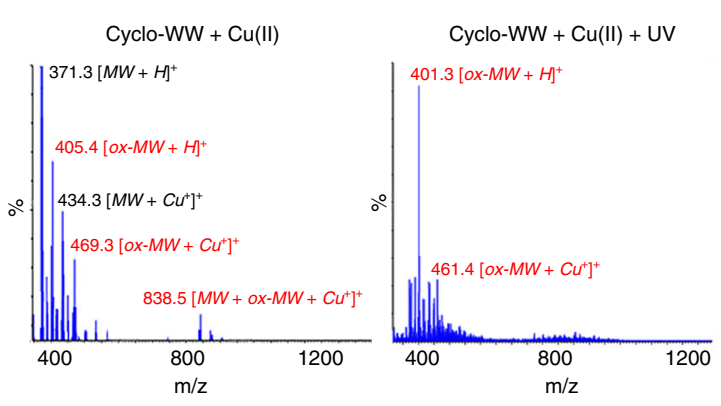

Fig. 4 Mechanisms underlying fluorescence modulation. a UV-Vis absorption spectra of cyclo-WW self-assemblies in the absence or presence of Zn(II) in $\mathrm{MeOH}$. A peak at $515 \mathrm{~nm}$ appeared when complexing cyclo-WW and $\mathrm{Zn}$ (II). b Job Plot analysis of cyclo-WW with Zn(II) at different ratios, with the total molar concentration fixed at $15.0 \mathrm{mM}$. The red lines were added for guideline, showing the intersection point at cyclo-WW proportion of 0.7. c Chemical shifts of cyclo-WW hydrogen atoms upon coordination with $\mathrm{Zn}(\mathrm{II})$, compared to the peptide alone. The hydrogen atoms in different chemical environments are marked with italicized letters and labeled in e. d FTIR spectra of cyclo-WW self-assemblies in the absence or presence of metal ion/UV irradiation. The peaks of the active bonds are numerically marked. Note that the IR spectra were vertically moved for clarity. e Schematic presentation showing the possible molecular mechanism of cyclo-WW dimer coordination with $\mathrm{Zn}(\mathrm{II})$ : the backbone diketopiperazine rings contribute to the complexation through nitrogen atoms, while the side-chain indole rings form aromatic interactions. $\mathbf{f ~ M S ~ s p e c t r a ~ o f ~ c y c l o - W W ~}+\mathrm{Cu}(\mathrm{II})$ and cyclo-WW + $\mathrm{Cu}(\mathrm{II})+\mathrm{UV}$, showing the MW of oxidized cyclo-WW (marked in red) and reduced $\mathrm{Cu}(\mathrm{I}$ ), confirming the redox reactions in the solutions

side-chain indole rings form $\pi-\pi$ interactions. It is possible that the complexation induces hindrance against further aggregation of the dimers, thus resulting in the dimers separated from each other and showing stable photoluminescence regardless of the excitation wavelength.

The larger size and aggregating nature of the cyclo- $\mathrm{WW}+\mathrm{Cu}$ (II) and cyclo-WW + UV nanospheres resulted in time-resolved parabolic evolution of the emission intensities (Supplementary Figs. 12, 13). In fact, the weak oxidation of $\mathrm{Cu}(\mathrm{II})$ induced slower aggregation dynamics, thus leading to a continuous increase of the fluorescent emission at $465 \mathrm{~nm}$, with an excitation of $370 \mathrm{~nm}$, persisting over one month (Supplementary Fig. 13a, black circles). This also resulted in larger aggregations with a long-wavelength $520 \mathrm{~nm}$ emission, following excitation at $395 \mathrm{~nm}$, constantly increasing over this period (Supplementary Fig. 13a, red circles).
As a control, the emission of cyclo-WW $+\mathrm{Zn}$ (II) decayed due to quenching (Supplementary Fig. 14). The redox reaction was verified by MS analysis, showing an $\mathrm{m} / z$ corresponding to the $\mathrm{MW}$ of oxidized cyclo-WW in the cyclo- $\mathrm{WW}+\mathrm{Cu}(\mathrm{II})$ solution, in addition to the dominant peak of native cyclo-WW (Fig. 4f, left panel). When combining both $\mathrm{Cu}(\mathrm{II})$ oxidation and $\mathrm{UV}$ irradiation (cyclo-WW $+\mathrm{Cu}(\mathrm{II})+\mathrm{UV})$, the synergistic effect dramatically accelerated the oxidation reaction, leading to an MS profile predominantly comprised of the $\mathrm{m} / \mathrm{z}$ of oxidized cyclo-WW (Fig. 4f, right panel). Additionally, in both massspectra, the MW of $\mathrm{Cu}(\mathrm{I})$-conjugated peptides was detected, indicating that the $\mathrm{Cu}$ (II) ions have been reduced. In contrast, in cyclo-WW $+\mathrm{Zn}(\mathrm{II})$, a chloride ion was always integrated, thus counteracting the positive charge of $\mathrm{Zn}$ (II) (Supplementary Fig. 15). 
The oxidation mechanism of $\mathrm{Cu}(\mathrm{II})$ was further verified using other metal ions with higher oxidative capability. When replacing $\mathrm{Cu}(\mathrm{II})$ with $\mathrm{Ag}(\mathrm{I})$ or $\left[\mathrm{AuCl}_{4}\right](-\mathrm{I})$, the sample solutions showed the same emission spectra with a maximum at $465 \mathrm{~nm}$ and bluegreen color under UV light (Supplementary Fig. 16a). Notably, the intense redox reduced the metal ions to elementary metals (Supplementary Fig. 16b). The different modulation mechanisms of $\mathrm{Zn}(\mathrm{II})$ and $\mathrm{Cu}$ (II) could also be confirmed by mixing with cyclo-FW, which did not complex with $\mathrm{Zn}$ (II) but showed enhanced fluorescence at $465 \mathrm{~nm}$ with a QY of $12 \%$ in the presence of $\mathrm{Cu}$ (II) (Supplementary Fig. 17).

The similar emission spectra of cyclo-WW $+\mathrm{Cu}(\mathrm{II})$ and cycloWW + UV suggested that the oxidized cyclo-WW did not complex with metal ions. In fact, cyclo-WW $+\mathrm{Zn}(\mathrm{II})+\mathrm{UV}$ had the same fluorescence emission as cyclo-WW + UV and cyclo$\mathrm{WW}+\mathrm{Cu}(\mathrm{II})+\mathrm{UV}$ (Supplementary Fig. 18), rather than that of cyclo-WW $+\mathrm{Zn}$ (II) (Fig. 2b). FTIR analysis demonstrated that in cyclo- $\mathrm{WW}+\mathrm{Cu}(\mathrm{II})$ and cyclo- $\mathrm{WW}+\mathrm{UV}$, the vibration of $\mathrm{N}-\mathrm{H}$ stretching of indole rings $\left(3393 \mathrm{~cm}^{-1}\right)$ significantly attenuated (Fig. 4d, peak 1) ${ }^{53}$, indicating that the redox took place at the $\mathrm{N}-\mathrm{H}$ bonds of the side-chains. Combined with the MS data, we propose that the two nitrogen atoms of the indole rings were conjugated with oxygen to form $-\mathrm{N}=\mathrm{O}$ bonds. In addition, the intensity of the ring breathing vibration $\left(742 \mathrm{~cm}^{-1}\right)$ also declined (Fig. $4 \mathrm{~d}$, peak 4$)^{53}$, suggesting that the redox severely disrupted the conformation of the rings ${ }^{54}$. It is speculated that the oxidation changed the electronic density and steric structures of the indole rings, thereby hindering the interactions of oxidized cyclo-WW with metal ions.

Near-infrared (NIR) fluorescence properties of the selfassemblies. A typical characteristic of QC materials is the dependence of their emission colors on particles $\operatorname{size}^{55}$. The excitation-dependent photoluminescent feature of the selfassemblies suggests that their emissions might be red-shifted to even longer wavelengths by introducing inhomogeneous size distributions ${ }^{40}$, for instance by using solvents that can facilitate the self-assembly. Indeed, when replacing $\mathrm{MeOH}$ with the more polar DMSO as a solvent for cyclo-WW $+\mathrm{Zn}(\mathrm{II})$, the extensive aggregation resulted in molecular excitation redshift, from $305 \mathrm{~nm}$ in $\mathrm{MeOH}$ to $310 \mathrm{~nm}$ (Supplementary Fig. 19), and the dimers self-assembled into larger spherical nanoparticles, $63.6 \pm 12.2 \mathrm{~nm}$ in diameter (Fig. 5a; Supplementary Figs. 20, 21). $\mathrm{X}$-ray diffraction analysis revealed distinct sharp peaks with high intensities (Supplementary Fig. 22), indicating the high crystallinity of the nanospheres, consistent with the crystallized nature of the QDs. These results illustrated the well-organized, periodical nano-lattice arrangement within the assemblies, thus confirming the directional organization of the dimeric QDs and the extensive internal QC effects.

Fluorescent characterization of the cyclo- $\mathrm{WW}+\mathrm{Zn}(\mathrm{II})$ nanospheres in DMSO demonstrated both visible and NIR fluorescence under a wide range of excitation wavelengths (Fig. 5b). Specifically, the peaks at $615 \mathrm{~nm}, 712 \mathrm{~nm}$, and $817 \mathrm{~nm}$ were emitted using excitation wavelengths between 520 and $780 \mathrm{~nm}$ (Fig. 5c; Supplementary Fig. 23), with lifetimes of 11.1 ns, $10.0 \mathrm{ns,}$ and 5.1 ns, respectively (Fig. 2f; Supplementary Fig. 8b), suggesting that as aggregation progressed, the excited electrons became more stable. This indicates a promising potential of the supramolecular structures to be used as bio-inspired alternatives for stable, long-term imaging. Correspondingly, the solution displayed distinct colors under different excitation wavelengths (Fig. 5d). The QYs were measured to be $18 \%$ and $27 \%$ for emissions at $712 \mathrm{~nm}$ and $817 \mathrm{~nm}$, respectively. Photobleaching evaluation experiments demonstrated that the photoluminescence of the nanospheres remained stable after continuous

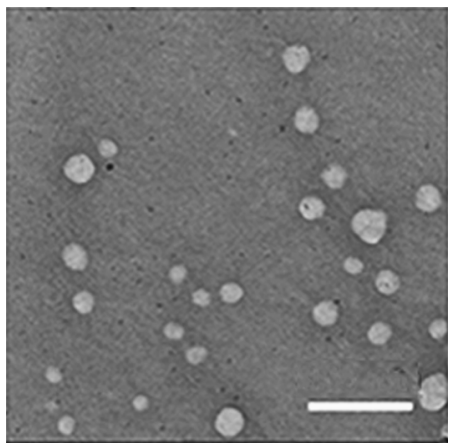

d

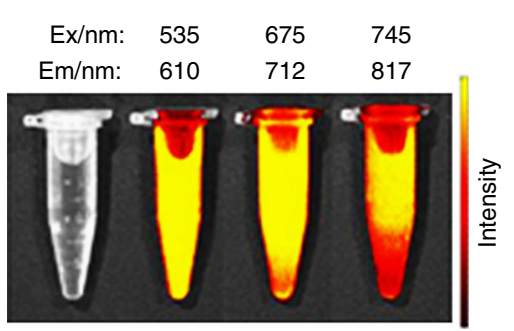

b

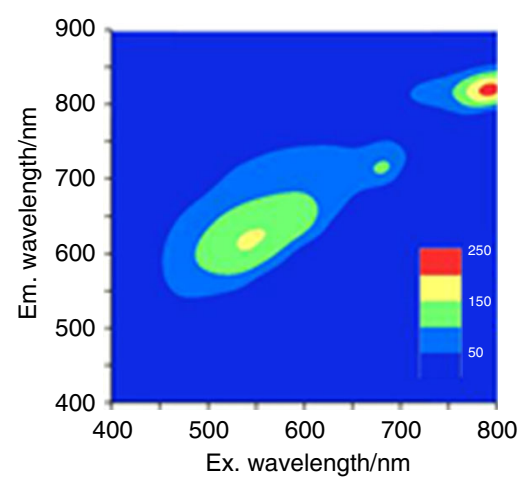

e

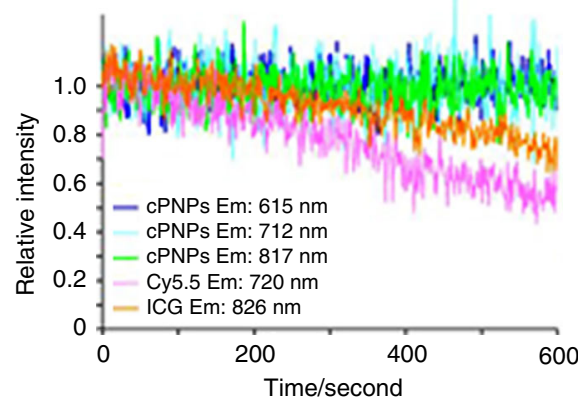

C

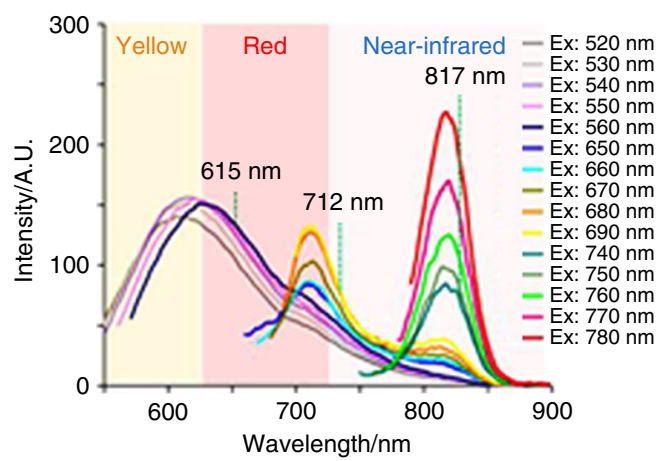

f

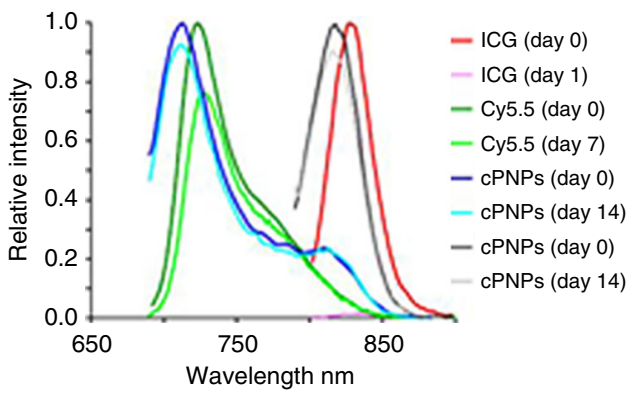

Fig. 5 NIR fluorescence properties of cyclo-WW $+\mathrm{Zn}(\mathrm{II})$ in DMSO. a TEM image of the self-assembled nanospheres. Scale bar: $300 \mathrm{~nm}$. $\mathbf{b}$ Emission vs. excitation profile of the nanospheres. c Extracted emission spectra of the nanospheres. $\mathbf{d}$ Visible to NIR photos of cyclo-WW $+\mathrm{Zn}(\mathrm{II}) \mathrm{DMSO}$ solution under various wavelengths. e Photobleaching and (f) photostability characterization of the cyclo-dipeptide nanoparticles (abbreviated as cPNPs) and of the fluorescent dyes ICG and Cy5.5, as controls 
irradiation for $600 \mathrm{~s}$, compared to the significant fluorescence decay observed for organic fluorescent dyes (indocyanine green (ICG) and cyanine 5.5 (Cy5.5)) (Fig. 5e). In addition, timeresolved characterization showed the fluorescence of the nanospheres to be stable even after exposure to natural light for two weeks, indicating their high photostability. In contrast, ICG lost all fluorescence after 1 day and Cy5.5 lost 20\% of the fluorescence after 1 week (Fig. 5f).

Application of the QC fluorescent self-assemblies. The photoluminescent nature endows the peptide QC self-assemblies the ability to be used for photo-stimulated devices, such as LEDs. By applying a mixture of dried cyclo-WW $+\mathrm{Zn}$ (II) dots in $\mathrm{MeOH}$ and polydimethylsiloxane (PDMS) onto an indium gallium nitride (InGaN) chip, a prototypical LED device using peptide self-assemblies as phosphors was fabricated (Fig. 6a). When applying voltages, bright green light was illuminated, as shown in the inset of Fig. 6a. Spectroscopic investigations demonstrated an emission around $550 \mathrm{~nm}$ regardless of the excitation wavelength (Fig. 6b), thus showing remarkable emission specificity. The red-shift of $30 \mathrm{~nm}$ from $520 \mathrm{~nm}$ (Fig. $2 \mathrm{~b}$ ) was probably due to aggregation that occurred during $\mathrm{MeOH}$ evaporation.

Furthermore, the bioinspired nature and the notable emission up to the NIR region suggest the potential utilization of cyclo$\mathrm{WW}+\mathrm{Zn}(\mathrm{II})$ nanospheres in biological systems. Based on an in vitro cytotoxicity analysis, the peptide nanoparticles showed good biocompatibility towards B16BL6 (murine melanoma cell line), MCF-7 (human breast cancer cell line), and $\mathrm{HaCaT}$ (human skin cell line) cells (Fig. 6c). Further, subcutaneous injection of the nanospheres into nude mice followed by NIR fluorescence imaging revealed distinct visible and NIR fluorescent signals at the injection site (Fig. 6d). Notably, the fluorescent signals were stable, showing no decay for one week, thus highlighting the possibility of utilizing the photoluminescent QC assemblies for in vivo bio-imaging applications. In addition, the advantage of easy modifications, such as specific targeting and controllable assembly/dis-assembly, facilitates simple functionalization of the assemblies ${ }^{56}$, thus exemplifying their promising potential for targeted therapy and controllable drug release.

Influence of aromatic side-chains. The important roles aromatic side-chains play during self-assembly suggest that the supramolecular morphologies and photoluminescent properties can be modulated by changing the aromatic moieties ${ }^{57}$. Therefore, three cyclo-dipeptides comprised of different aromatic amino acids, i.e., cyclo-dihistidine (cyclo-HH), cyclo-diphenylalanine (cyclo-FF), and cyclo-dityrosine (cyclo-YY) (Fig. 7a), were examined under the same conditions. Morphological characterization showed that the cyclo-dipeptides self-assembled into diverse supramolecular structures. Specifically, cyclo-HH formed nanofibers in $\mathrm{MeOH}$ (Fig. 7b), cyclo-FF formed spherical nanoparticles in DMSO (Fig. 7c), and cyclo-YY assembled into nanorods in DMSO (Fig. 7d). AFM analysis demonstrated small aggregates less than $4 \mathrm{~nm}$ in height at a lower concentration $(0.5 \mathrm{mM})$, with a

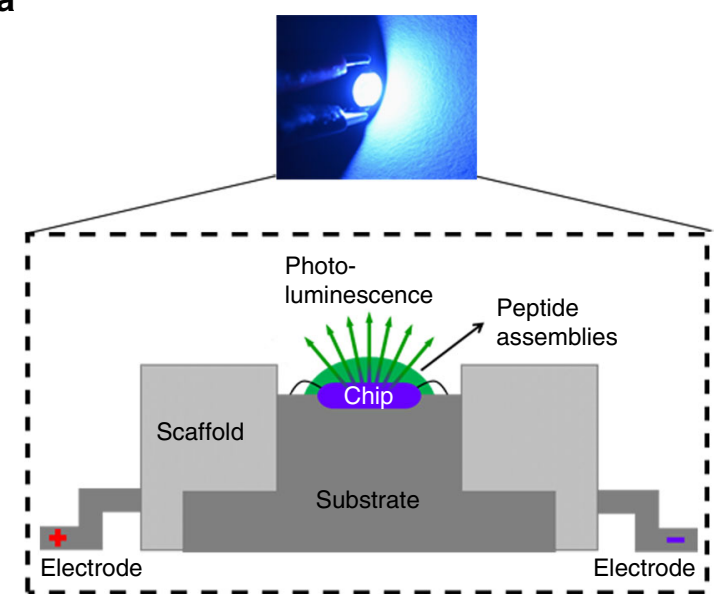

C

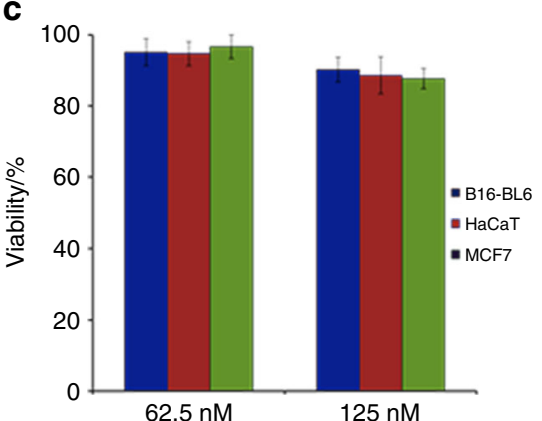

d b
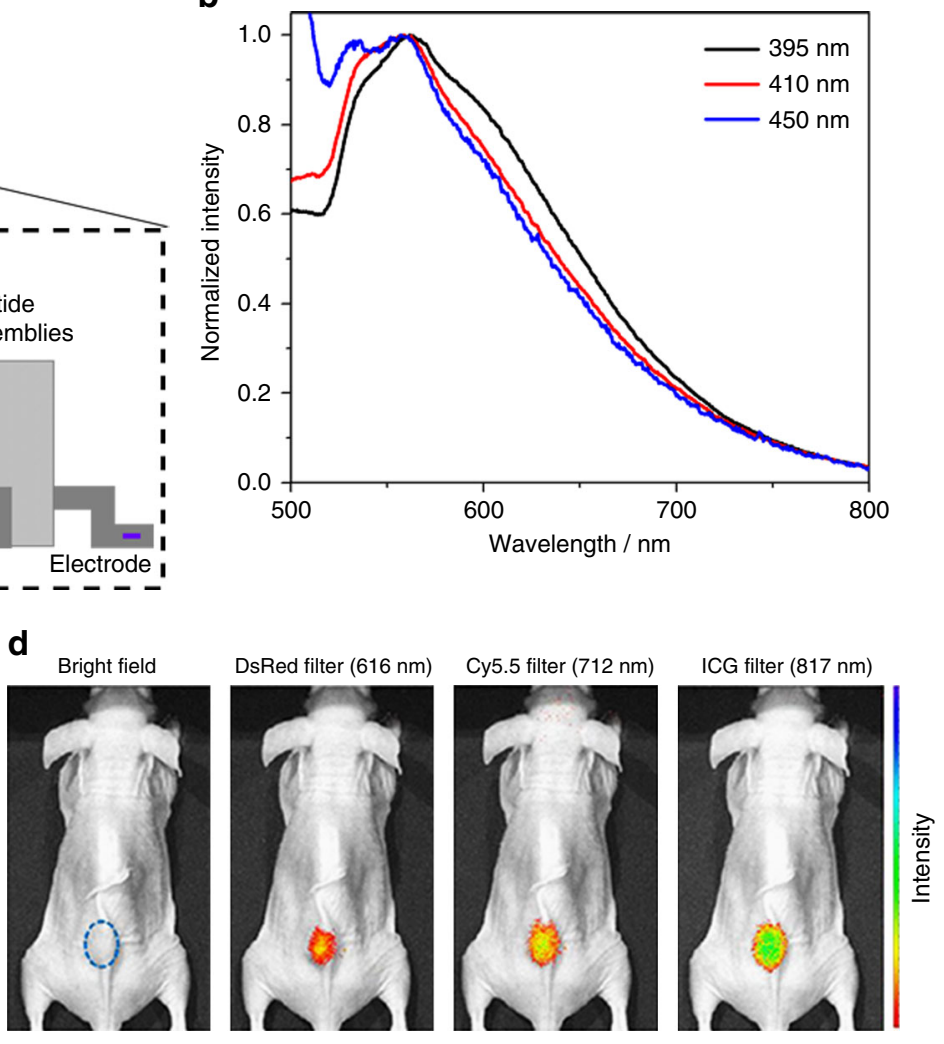

Fig. 6 Application of the cyclo-WW $+\mathrm{Zn}$ (II) QC self-assemblies. a Schematic presentation of the LED setup using dried cyclo-WW $+\mathrm{Zn}$ (II) assembled in $\mathrm{MeOH}$ as phosphors. The upper inset shows the working depiction of a prototype, emitting bright green light (ex: 450 nm). b Spectroscopic characterization of the LED photoluminescence using three excitation wavelengths, as indicated, showing the same emission at 550 nm. c Cytotoxicity test of the cyclo-WW + Zn(II) nanospheres in DMSO (62.5 nM and $125 \mathrm{nM}$ ) towards B16-BL6, HaCaT, and MCF7 cells. Viability relative to untreated controls $\pm \mathrm{sd}$, designated as error bars, is shown based on three repeats and averaged. d In vivo whole body NIR fluorescent imaging following subcutaneous injection of the nanospheres $(50 \mu \mathrm{L}, 2.7 \mathrm{mM})$ into nude mice, showing notable emissions under various excitations. The dotted circle indicates the location of injection 
a<smiles>O=C(O)C[C@H]1NC(=O)[C@H](Cc2c[nH]cn2)NC1=O</smiles>

b

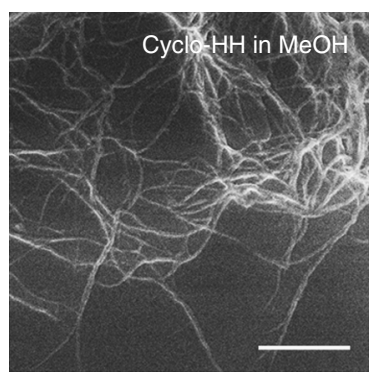

e

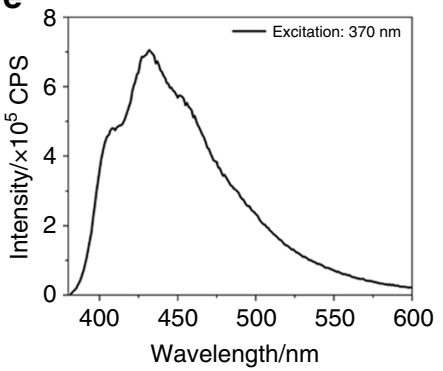<smiles>O=C1N[C@H](Cc2ccccc2)C(=O)N[C@@H]1Cc1ccccc1</smiles>

C

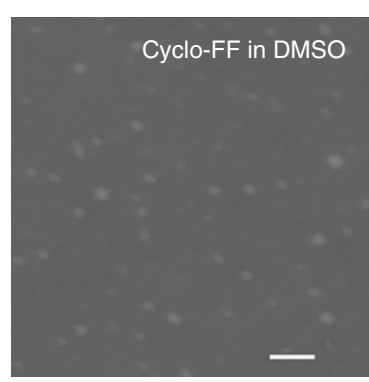

\section{$f$}

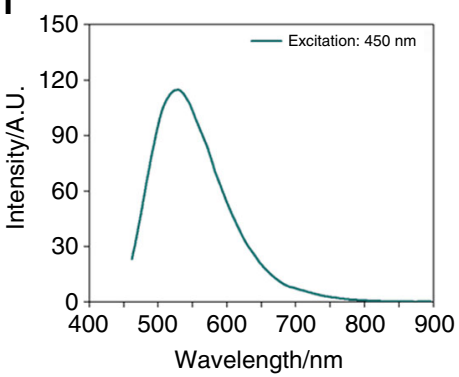

Cyclo-YY<smiles>O=C1NC(Cc2ccc(O)cc2)C(=O)NC1Cc1ccc(O)cc1</smiles>

d

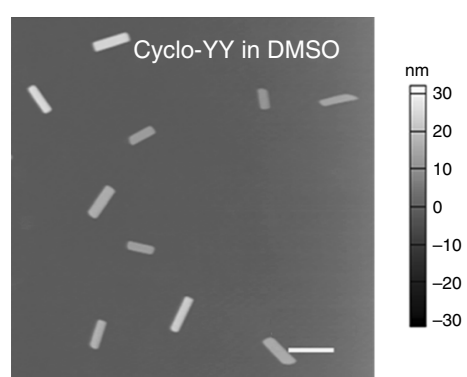

g

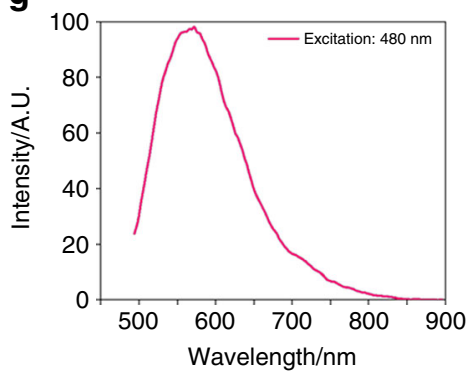

Fig. 7 The effect of diverse aromatic side-chains. a Molecular structures of cyclo-HH, cyclo-FF, and cyclo-YY. b-d Microscopic images showing the self-assemblies of (b) cyclo-HH in MeOH (scale bar: $1 \mu \mathrm{m}$ ), (c) cyclo-FF in DMSO (scale bar: $50 \mathrm{~nm}$ ), and (d) cyclo-YY in DMSO (scale bar: $500 \mathrm{~nm}$ ). e-g Fluorescent emission of (e) cyclo-HH in $\mathrm{MeOH}$, (f) cyclo-FF in DMSO, and (g) cyclo-YY in DMSO

dots for cyclo-HH, cyclo-FF, and thin nanofibers for cyclo-YY (Supplementary Fig. 24). This suggested that like cyclo-FW and cyclo-WW, the self-assemblies of these three cyclo-dipeptides were also composed of QC intermediates. Fluorescent characterization demonstrated that the distinct morphologies presented different photoluminescence, with cyclo-HH nanofibers showing emission at $430 \mathrm{~nm}$ (Ex: $370 \mathrm{~nm}$ ) (Fig. 7e), cyclo-FF nanoparticles at $530 \mathrm{~nm}$ (Ex: $450 \mathrm{~nm})$ (Fig. $7 \mathrm{f}$ ), and cyclo-YY nanorods at $570 \mathrm{~nm}(\mathrm{Ex}: 480 \mathrm{~nm})($ Fig. $7 \mathrm{~g})$.

\section{Discussion}

We demonstrate here that $\mathrm{W}$-based aromatic cyclo-dipeptides self-assemble to dimeric QDs, which act as building blocks to further organize into supramolecular structures. Due to the extensive and directional QC regions within, the assemblies show intrinsic photoluminescence properties. Especially, through amino acid substitution, metal ion coordination, molecular oxidation or UV irradiation, and solvent replacement, the supramolecular morphologies could be finely controlled, ranging from dimeric QDs to larger organizations. Correspondingly, the photoluminescence could be tuned, covering most of the visible into the NIR spectral region. The biocompatibility and wide-spectrum emission features make these supramolecular structures highly suitable for in vivo bio-imaging applications with no detected cytotoxicity and for the fabrication of LEDs, where the assemblies are used as phosphors. Finally, further exploration is expected to demonstrate the potential of these self-assemblies for diverse applications. Thus, a variety of modulation strategies, such as the design of larger cyclo-oligopeptides (cyclo-tripeptides, cyclo-tetrapeptides, etc.), flexible assembly approaches (co-assembly, covalent conjugation), introduction of more complicated reactions and self-assembly under different conditions (using physical vapor deposition), can be employed to tune the selfassembly and photoluminescence of cyclo-peptides.

Metal ions play important roles in peptide self-assembly processes. The cyclo-dipeptides QC self-assemblies described here might be used as fluorescent markers to probe the dynamics of aggregations processes, such as amyloidogenic polypeptide folding. This can supply a real-time, visual detection of the metabolic activities $^{58}$, and even facilitate screening for inhibitors of these processes, acting by interaction with metal ions. In addition, their nanoscale sizes, opto-electric properties, and intrinsic biocompatibility allow these nanostructures to be implanted into neuronal cells in order to investigate the interface between the structures and neurons ${ }^{59}$. Therefore, the metabolic procedures of the QC assemblies in neural cells and their response and influence on neuronal activities (such as current formation, synaptic activities, and signal transduction) can be studied by tracking their signals. This can provide the basis for diagnosis and treatment of sensory functions.

To conclude, based on comprehensive analyses of aromatic cyclo-dipeptides self-assemblies, as presented here, these materials could serve as bioinspired, organic, supramolecular alternatives to complement the state-of-the-art inorganic counterparts, and further broaden the application of QC materials.

\section{Methods}

Cells lines. The MCF-7 human breast cancer cell line was purchased from the American Type Culture Collection (ATCC). The B16-BL6 murine melanoma cell line was obtained from the National Cancer Institute-Central Repository. The human skin HaCaT keratinocyte cell line, a transformed human epidermal cell line, was obtained from Dr. Norbert Fusenig of the Germany Cancer Research Center. 
MCF-7 cells were cultured in Eagle's Minimum Essential Medium supplemented with 10\% FBS. B16-BL6 and HaCaT cells were cultured in Dulbecco's Modified Eagle's Medium supplemented with $10 \%$ FBS. All culture media contained $1 \%$ penicillin and cells were maintained at $37^{\circ} \mathrm{C}$ in a humidified $5 \% \mathrm{CO}_{2}$ incubator Cells were periodically examined to verify the absence of mycoplasma contamination using the commercial detection kit (Lonza, Switzerland, LT07-703).

Mice and care. Athymic male NU/NU nude mice (6-week old) were purchased from Charles River (Wilmington, MA, USA) and maintained on a 16:8 h light-dark cycle. All procedures for animal use were approved by the Institutional Animal Care and Use Committee (IACUC) at The Ohio State University.

Materials. Cyclo-FW, cyclo-WW, cyclo-YY, and cyclo-FF were purchased from Bachem (Bubendorf, Switzerland), cyclo-F $w$ and cyclo-HH from DGpeptides (Hangzhou, China), anhydrous zinc chloride $\left(\mathrm{ZnCl}_{2}\right)$, copper chloride dihydrate $\left(\mathrm{CuCl}_{2} \cdot 2 \mathrm{H}_{2} \mathrm{O}\right)$, and anhydrous $\mathrm{MeOH}$ from Sigma Aldrich (Rehovot, Israel), DMSO from Sigma-Aldrich (St. Louis, MO). All materials were used as received without further purification. Water was processed using a Millipore purification system (Darmstadt, Germany) with a minimum resistivity of $18.2 \mathrm{M} \Omega \mathrm{cm}$.

Sample preparation. Cyclo-dipeptides were added to anhydrous $\mathrm{MeOH}, \mathrm{MeOH}$ solutions of $\mathrm{ZnCl}_{2}\left(\mathrm{CuCl}_{2}\right)$, or DMSO solution of $\mathrm{ZnCl}_{2}$, to final concentrations of $5.0 \mathrm{mM}$ cyclo-dipeptides and $10.0 \mathrm{mM}$ metal salts. To dissolve the peptides, the solutions were incubated in a water bath at $70^{\circ} \mathrm{C}$ for $5 \mathrm{~min}$, after which the solutions became transparent.

Fluorescence. UV-Visible fluorescence characterization: $600 \mu \mathrm{L}$ sample solution was pipetted into a $1.0 \mathrm{~cm}$ path-length quartz cuvette, and the spectrum was collected using a FluoroMax-4 Spectrofluorometer (Horiba Jobin Yvon, Kyoto, Japan) at ambient temperature. For molecular fluorescence, the emission wavelength was set at $350 \mathrm{~nm}$ with a slit of $3 \mathrm{~nm}$, and the excitation wavelength was set at 200-330 $\mathrm{nm}$ with a slit of $3 \mathrm{~nm}$. For self-assembly fluorescence, the excitation wavelength was set at $370 \mathrm{~nm}$ with a slit of $3 \mathrm{~nm}$, and the emission wavelength was set at $380-700 \mathrm{~nm}$ with a slit of $3 \mathrm{~nm}$. For excitation-dependent maximal emission evolution experiment, the excitation wavelengths were set at $300-450 \mathrm{~nm}$ with a slit of $3 \mathrm{~nm}$, and the emission wavelengths were set at $380-600 \mathrm{~nm}$ with a slit of $3 \mathrm{~nm}$. According to the samples, anhydrous $\mathrm{MeOH}$ or $\mathrm{MeOH}$ solution of $\mathrm{ZnCl}_{2}\left(\mathrm{CuCl}_{2}\right)$ was used as background and subtracted. At least five measurements were performed and averaged for accuracy.

NIR fluorescence characterization: Cary Eclipse Fluorescence Spectrophotometer (Agilent Technologies) was used for measuring fluorescence excitation and emission spectra, with both slits width set at $5 \mathrm{~nm}$. Three measurements were performed and averaged for accuracy.

Fluorescent decay measurement (lifetime). Six hundred microliter sample solution was pipetted into a $1.0 \mathrm{~cm}$ path-length quartz cuvette, and the spectrum was collected using a FluoroMax-4 Spectrofluorometer (Horiba Jobin Yvon, Kyoto, Japan) equipped with a NanoLED laser excitation source at ambient temperature. The wavelength was set as the maximum excitation and emission of the samples, and a LUDOX sample (silica beads, $2 \mu \mathrm{m}$ ) was used as the prompt. The lifetime was determined by fitting the fluorescent decay data from the DAS6 Analysis software (Horiba Jobin Yvon, Kyoto, Japan). Three measurements were performed and averaged for accuracy.

Fluorescence photobleaching. The kinetic measurements of Cy5.5 (excitation: $650 \mathrm{~nm}$, emission: $720 \mathrm{~nm}$ ), ICG (excitation: $785 \mathrm{~nm}$, emission: $826 \mathrm{~nm}$ ) and cycloWW + Zn(II) in DMSO (excitation: 550/680/770 nm, emission: 615/712/817 nm) were conducted using a Cary Eclipse Fluorescence Spectrophotometer (Agilent Technologies). The fluorescence emission data was collected at 80 points per second for $10 \mathrm{~min}$. The excitation and emission slit widths were each set at 5 $\mathrm{nm}$. At least three measurements were performed and averaged for accuracy.

Fluorescence photostability. Fluorescence emission spectra were measured at different time points ( 1 day, 7 days, and 14 days). The excitation and emission slit widths were each set at $5 \mathrm{~nm}$. Three measurements were performed and averaged for accuracy.

UV-Vis absorption. For cyclo-dipeptides solutions absorption, $45 \mu \mathrm{L}$ of the sample solution was pipetted into a 96-well UV-Star UV transparent plate (Greiner BioOne, Frickenhausen, Germany), and the UV-Vis absorbance was recorded using a Biotek Synergy HT plate reader (Biotek, Winooski, VT, USA), with a normal reading speed and calibration before reading. Anhydrous $\mathrm{MeOH}$ was used as background and subtracted. Three measurements were performed and averaged for accuracy.
QD radius calculation. The QD radius was calculated based on the model of organic QDs ${ }^{34}$, as shown in Eq. 1:

$$
R=\pi r_{\mathrm{B}}^{0} \sqrt{\frac{m_{0} / M}{\frac{\mu}{m_{0} \varepsilon_{\infty}^{2}}-\frac{E_{\varepsilon x}^{\mathrm{QD}}}{R_{y}}}}
$$

Where $r_{\mathrm{B}}^{0}=\hbar^{2} / m_{0} \mathrm{e}^{2}=0.53 \AA$ is the Bohr radius of the hydrogen atom; $m_{0}$ is the free electron mass $\left(9.11 \times 10^{-31} \mathrm{~kg}\right) ; R_{y}=m_{0} \mathrm{e}^{4} / 2 \hbar^{2}=13.56 \mathrm{eV}$ is the Rydberg constant; $M=m_{\mathrm{e}}+m_{\mathrm{h}}$ is the translation mass of the exciton $\left(m_{\mathrm{e}}\right.$ and $m_{\mathrm{h}}$ are the effective mass of electron and hole, respectively); $\mu=m_{\mathrm{e}} m_{\mathrm{h}}\left(m_{\mathrm{e}}+m_{\mathrm{h}}\right)$ is the reduced exciton mass; $\varepsilon_{\infty}$ is the high-frequency dielectric constant of the QD; $E_{\mathrm{ex}}^{\mathrm{QD}}$ is the exciton binding energy.

Since obtaining the accurate reflective index of peptide QDs is technically challenging, the refractive index of the analogous benzene crystal, namely $n=1.5$, was used to calculate $\varepsilon_{\infty}$. This defines $\varepsilon_{\infty}=n^{2}=2.25$.

The optical absorption starts from $\lambda_{\text {ion }} 250 \mathrm{~nm}\left(\hbar \omega_{\text {ion }}=4.96 \mathrm{eV}\right)$ (Fig. 1c), corresponding to the breaking of the binding exciton state. The value of $\hbar \omega_{\text {ion }}$ corresponds to the QD energy gap. The difference between $\hbar \omega_{\text {ion }}$ and the phononless line $\hbar \omega_{\mathrm{g}}^{0}=4.59 \mathrm{eV}$ equals $0.37 \mathrm{eV}$, representing the exciton binding energy, $E_{\mathrm{ex}}^{\mathrm{QD}}$ of the $\mathrm{QD}$.

The effective masses of electrons and holes are almost identical and close to $0.5 m_{0}$. Consequently, for $\mu=0.5 m_{\mathrm{e}}=0.25 m_{0}$ and $M=M_{0}$, the QD diameter of the cyclo-dipeptides was calculated to be $\mathrm{D} \approx 2.24 \mathrm{~nm}$, approximately the dimension of a dimer.

For Job Plot analysis of cyclo-WW $+\mathrm{Zn}(\mathrm{II})$, a fixed total concentration of $15.0 \mathrm{mM}$ was used, with the following molar proportions (corresponding concentrations) of cyclo-WW: $0.0(0.0 \mathrm{mM}), 0.1(1.5 \mathrm{mM}), 0.2(3.0 \mathrm{mM}), 0.3$ $(4.5 \mathrm{mM}), 0.4(6.0 \mathrm{mM}), 0.5(7.5 \mathrm{mM}), 0.6(9.0 \mathrm{mM}), 0.7(10.5 \mathrm{mM}), 0.8(12.0 \mathrm{mM})$, $0.9(13.5 \mathrm{mM}), 1.0(15.0 \mathrm{mM})$. One milliliter sample of each solution was pipetted into a $1.0 \mathrm{~cm}$ path-length quartz cuvette, and a T60 visible spectrophotometer (PG Instruments, Leicestershire, United Kingdom) was used for spectra collection with a fixed spectral bandwidth of $2 \mathrm{~nm}$ and a $200-800 \mathrm{~nm}$ wavelength range. Anhydrous $\mathrm{MeOH}$ solutions of $\mathrm{ZnCl}_{2}$ at the corresponding concentrations were used as background and subtracted. The absorbance at $515 \mathrm{~nm}$ was extracted to generate the Job Plot vs. molar proportions of cyclo-WW.

\section{Theoretical calculations of molecular orbital amplitudes and energy levels.} Density functional theory calculations were carried out based on the self-consistent solution of Kohn-Sham function and the projector augmented wave pseudopotential as implemented in Vienna Ab-initio Simulation Package (VASP). The exchange-correlation potential is in the form of Perdew-Burke-Ernerhof (PBE) with generalized gradient approximation (GGA). For the structural relaxation, the energy convergence threshold was set to $10^{-5} \mathrm{eV}$ and the residual force on each atom was less than $0.03 \mathrm{eV}^{-1}$. The cutoff energy for the plane-wave basis was set to $500 \mathrm{eV}$. To eliminate interaction between the molecule and its periodic images, a vacuum distance larger than $15 \AA$ for each direction in the supercell geometry was used.

Nucleic magnetic resonance (NMR). Cyclo-WW or cyclo-WW $+\mathrm{Zn}$ (II) were dissolved in deuterated solvent with tetramethylsilane as the internal standard to prepare sample solutions with $5.0 \mathrm{mM}$ dipeptide and $10.0 \mathrm{mM} \mathrm{Zn}(\mathrm{II}) .{ }^{1} \mathrm{H}$ NMR spectra were recorded on a Bruker AV-400 NMR spectrometer with chemical shifts reported as p.p.m. The difference in the chemical shifts values before and after the addition of $\mathrm{Zn}$ (II) into the cyclo-WW solution $\left(\Delta \delta=\left(\delta_{\text {(cyclo-WW) }}-\delta_{\text {(cyclo-WW }+\mathrm{Zn}}\right.\right.$ (II))) were calculated in ppb and plotted as a function of amide, aromatic, and aliphatic protons.

Mass spectrometry (MS). The MS experiment was performed using a LCMS Xevo-TQD system including an Acquity model UPLC and a triple quad mass spectrometer (Waters, Massachusetts, USA). The positive electrospray ionization (ES+) channel was used for analysis.

Scanning electron microscopy (SEM). Twenty microliter solution samples were placed onto a clean glass slide and allowed to adsorb for a few seconds. After removing excessive liquid with filter paper, the slide was coated with $\mathrm{Cr}$ and observed under a JSM-6700 field emission scanning electron microscope (JEOL, Tokyo, Japan) operated at $10 \mathrm{kV}$.

Quantum yield (QY) measurement. Five milliliter sample solution was pipetted into a $1.0 \mathrm{~cm}$ path-length quartz cuvette with a tube (Hellma, Müllheim, Germany) and the absolute quantum yield was measured on an absolute PL quantum yield spectrometer C11347 (Hamamatsu Photonics, Shizuoka, Japan) at ambient temperature. The relevant blanks, namely anhydrous $\mathrm{MeOH}, \mathrm{MeOH}$ solutions of $\mathrm{ZnCl}_{2}\left(\mathrm{CuCl}_{2}\right)$, or DMSO solution of $\mathrm{ZnCl}_{2}$, were used as background and subtracted. At least five measurements were performed and averaged for accuracy. 
Dynamic light scattering (DLS). Eight hundred and fifty microliter of the sample solution was introduced into a DTS1070 folded capillary cell (Malvern, Worcestershire, U.K.), and the size was measured using a Zetasizer Nano ZS analyzer (Malvern Instruments, Malvern, UK) at $25.0^{\circ} \mathrm{C}$ and a backscatter detector $\left(173^{\circ}\right)$. Three measurements were performed and averaged for accuracy.

Fourier transform infrared spectroscopy (FTIR). Seven hundered and fifty microliter of the sample solution was dropped onto polysthylene IR card (International Crystal Labs, Garfield, New Jersey, USA) and air drying. The FTIR spectra were recorded on a Nicolet 6700 FTIR spectrometer (Thermo Scientific, Waltham, Massachusetts, USA), from 4000 to $400 \mathrm{~cm}^{-1}$ at room temperature. One hundred and twenty-eight scans were collected with a spectral resolution of $4 \mathrm{~cm}^{-1}$ in nitrogen atmosphere. Corresponding reference spectra (anhydrous $\mathrm{MeOH}$ or $\mathrm{MeOH}$ solution of $\mathrm{ZnCl}_{2}\left(\mathrm{CuCl}_{2}\right)$ ) were recorded under identical conditions and subtracted.

Atomic force microscopy (AFM). The cyclo-dipeptide self-assemblies were characterized using an MFP-3D AFM system with an ACTA-50 Probe (AppNano). Pyramidal shaped silicon tip was used, with radius of curvature below $10 \mathrm{~nm}$, alumina reflex coating, a spring constant of $13-77 \mathrm{~N} \mathrm{~m}^{-1}$, and a frequency of $200-400 \mathrm{kHz}$. The Igor Pro software in AC mode was used for image recording, thereby minimizing the distortion caused by mechanical interactions between the surface and the AFM tip.

Transmission electron microscopy (TEM). A carbon-coated copper grid was placed on a $10 \mu \mathrm{L}$ cyclo-dipeptide droplet for $1 \mathrm{~min}$ and then blotted. Next, the washed grid was placed on a $10 \mu \mathrm{L}$ droplet containing $4 \%(\mathrm{w} / \mathrm{v})$ uranyl acetate solution for $1 \mathrm{~min}$ and then blotted. Samples were examined using Tecnai G2 Spirit TEM (FEI) at $80 \mathrm{kV}$.

X-ray diffraction (XRD). Spectra were recorded using a Bruker D8 Advance X-ray powder diffractometer (Bruker) at room temperature with a scan range $2 \theta$ of $5-45^{\circ}$ and a count of two seconds. Cyclo-WW $+\mathrm{Zn}$ (II) nanospheres powder was placed on the standard flat sample reflection holder. Data collection and analysis was performed using the MDI Jade software.

Fabrication and characterization of LEDs. Commercially available InGaN chips were used at the bottom of the LED base. For the preparation of the color conversionlayer, the cyclo-WW $+\mathrm{Zn}$ (II) $\mathrm{MeOH}$ solution was purged dry using ultrapure nitrogen, and then mixed with PDMS at a mass ratio of 1:1. The mixtures were applied on InGaN chips and after curing at $80^{\circ} \mathrm{C}$ for $1 \mathrm{~h}$, the LEDs peptides phosphors were obtained.

Cytotoxicity. HaCaT, B16-BL6, and MCF-7 cells were seeded in a 96-well plate with $100 \mu \mathrm{L}$ of culture medium per well and incubated at $37^{\circ} \mathrm{C}$ in $5 \% \mathrm{CO}_{2}$ for $12 \mathrm{~h}$ to allow the cells to adhere to the surface. The medium was then replaced with a medium containing cyclo-WW $+\mathrm{Zn}(\mathrm{II})$ nanospheres at two different concentrations $(62.5,125.0 \mathrm{nM}$ in cell culture medium), or with naive medium as a control. Cells were incubated for $24 \mathrm{~h}$ before determining cell viability using the CCK- 8 assay (Dojindo Molecular Technologies), according to the manufacturer's instructions. The absorbance at $490 \mathrm{~nm}$ was determined using Opsys microplate reader (Dynex Technologies, Chantilly, VA).

In vivo NIR imaging. Cyclo- $\mathrm{WW}+\mathrm{Zn}(\mathrm{II})$ nanospheres $(50 \mu \mathrm{L}, 2.7 \mathrm{mM}$ diluted in water) were administered into nude mice by subcutaneous injection. Whole body NIR fluorescence imaging was conducted with the mice anesthetized (2.5\% isoflurane in oxygen flow, $1.5 \mathrm{~L} \mathrm{~min}^{-1}$ ) immediately following the injection using an IVIS Spectrum Imaging System (PerkinElmer). All images were taken using emission filters designed for DsRed (575-650 nm), Cy5.5 (695-770 nm), and ICG (810-875 $\mathrm{nm}$ ), with excitation wavelengths of $535 \mathrm{~nm}, 640 \mathrm{~nm}$, and $745 \mathrm{~nm}$, respectively. The fluorescent light emitted from the mice was detected by a CCD camera. Data acquisition and analysis was performed using the Living Image 4.2.1 software.

Data availability. The authors declare that the data supporting the findings of this study are available within the article and its Supplementary Information file, or are available from the authors upon request.

Received: 12 February 2018 Accepted: 10 July 2018

Published online: 13 August 2018

\section{References}

1. Gao, X. H., Cui, Y. Y., Levenson, R. M., Chung, L. W. K. \& Nie, S. M. In vivo cancer targeting and imaging with semiconductor quantum dots. Nat. Biotechnol. 22, 969-976 (2004).
2. Michalet, $X$. et al. Quantum dots for live cells, in vivo imaging, and diagnostics. Science 307, 538-544 (2005).

3. Larson, D. R. et al. Water-soluble quantum dots for multiphoton fluorescence imaging in vivo. Science 300, 1434-1436 (2003).

4. Lim, X. Z. The nanoscale rainbow. Nature 531, 26-28 (2016).

5. Derfus, A. M., Chan, W. C. \& Bhatia, S. N. Probing the cytotoxicity of semiconductor quantum dots. Nano. Lett. 4, 11-18 (2004).

6. Hardman, R. A toxicologic review of quantum dots: toxicity depends on physicochemical and environmental factors. Environ. Health Perspect. 114, 165-172 (2006).

7. Resch-Genger, U., Grabolle, M., Cavaliere-Jaricot, S., Nitschke, R. \& Nann, T. Quantum dots versus organic dyes as fluorescent labels. Nat. Methods 5, 763-775 (2008).

8. Aida, T., Meijer, E. W. \& Stupp, S. I. Functional supramolecular polymers. Science 335, 813-817 (2012).

9. Hauser, C. A. E. \& Zhang, S. G. Nanotechnology: peptides as biological semiconductors. Nature 468, 516-517 (2010).

10. Mei, J. et al. Aggregation-induced emission: the whole is more brilliant than the parts. Adv. Mater. 26, 5429-5479 (2014).

11. Reches, M. \& Gazit, E. Casting metal nanowires within discrete self-assembled peptide nanotubes. Science 300, 625-627 (2003).

12. Tao, K., Makam, P., Aizen, R. \& Gazit, E. Self-assembling peptide semiconductors. Science 358, eaam9756 (2017).

13. Zhang, S. G. Fabrication of novel biomaterials through molecular selfassembly. Nat. Biotechnol. 21, 1171-1178 (2003).

14. Yan, X. H., Li, J. B. \& Möhwald, H. Self-assembly of hexagonal peptide microtubes and their optical waveguiding. Adv. Mater. 23, 2796-2801 (2011)

15. Amdursky, N. et al. Blue luminescence based on quantum confinement at peptide nanotubes. Nano. Lett. 9, 3111-3115 (2009).

16. Lee, J. S. et al. Self-assembly of semiconducting photoluminescent peptide nanowires in the vapor phase. Angew. Chem. Int. Ed. 50, 1164-1167 (2011),

17. Gan, Z. X., Wu, X. L., Zhu, X. B. \& Shen, J. C. Light-induced ferroelectricity in bioinspired self-assembled diphenylalanine nanotubes/microtubes. Angew. Chem. Int. Ed. 52, 2055-2059 (2013).

18. Nguyen, V., Zhu, R., Jenkins, K. \& Yang, R. Self-assembly of diphenylalanine peptide with controlled polarization for power generation. Nat. Commun. 7 , 13566 (2016).

19. Esin, A. et al. Pyroelectric effect and polarization instability in self-assembled diphenylalanine microtubes. Appl. Phys. Lett. 109, 142902 (2016).

20. Kim, S. J., Kim, J. H., Lee, J. S. \& Park, C. B. Beta-sheet-forming, selfassembled peptide nanomaterials towards optical, energy, and healthcare applications. Small 11, 3623-3640 (2015).

21. Adler-Abramovich, L. \& Gazit, E. The physical properties of supramolecular peptide assemblies: from building block association to technological applications. Chem. Soc. Rev. 43, 6881-6893 (2014).

22. Yan, X. H., Zhu, P. L. \& Li, J. B. Self-assembly and application of diphenylalanine-based nanostructures. Chem. Soc. Rev. 39, 1877-1890 (2010).

23. Lampel, A. et al. Polymeric peptide pigments with sequence-encoded properties. Science 356, 1064-1068 (2017).

24. Fan, Z., Sun, L. M., Huang, Y. J., Wang, Y. Z. \& Zhang, M. J. Bioinspired fluorescent dipeptide nanoparticles for targeted cancer cell imaging and realtime monitoring of drug release. Nat. Nanotechnol. 11, 388-394 (2016).

25. Nalluri, S. K. M. et al. Biocatalytic self-assembly of supramolecular chargetransfer nanostructures based on n-type semiconductor-appended peptides. Angew. Chem. Int. Ed. 53, 5882-5887 (2014).

26. Ryu, J., Lim, S. Y. \& Park, C. B. Photoluminescent peptide nanotubes. Adv. Mater. 21, 1577-1581 (2009).

27. Sone, E. D. \& Stupp, S. I. Semiconductor-encapsulated peptide-amphiphile nanofibers. J. Am. Chem. Soc. 126, 12756-12757 (2004).

28. Akdim, B., Pachter, R. \& Naik, R. R. Self-assembled peptide nanotubes as electronic materials: an evaluation from first-principles calculations. Appl. Phys. Lett. 106, 183707 (2015).

29. Amdursky, N. Enhanced solid-state electron transport via tryptophan containing peptide networks. Phys. Chem. Chem. Phys. 15, 13479-13482 (2013).

30. Yan, X. H., Su, Y., Li, J. B., Früh, J. \& Möhwald, H. Uniaxially oriented peptide crystals for active optical waveguiding. Angew. Chem. Int. Ed. 50, 11186-11191 (2011)

31. Manchineella, S. \& Govindaraju, T. Molecular self-assembly of cyclic dipeptide derivatives and their applications. ChemPlusChem 82, 88-106 (2016).

32. Amdursky, N. et al. Structural transition in peptide nanotubes. Biomacromolecules 12, 1349-1354 (2011).

33. Gazit, E. Peptide nanostructures: aromatic dipeptides light up. Nat. Nanotechnol. 11, 309-310 (2016).

34. Amdursky, N., Molotskii, M., Gazit, E. \& Rosenman, G. Elementary building blocks of self-assembled peptide nanotubes. J. Am. Chem. Soc. 132, 15632-15636 (2010) 
35. Amdursky, N., Molotskii, M., Gazit, E. \& Rosenman, G. Self-assembled bioinspired quantum dots: optical properties. Appl. Phys. Lett. 94, 261907 (2009).

36. Maia, F. F. Jr. et al. Anhydrous crystals of DNA bases are wide gap semiconductors. J. Chem. Phys. 134, 175101 (2011).

37. Zanatta, G. et al. L-asparagine crystals with wide gap semiconductor features: optical absorption measurements and density functional theory computations. J. Chem. Phys. 140, 124511 (2014).

38. Zhang, H. K. et al. Why do simple molecules with "isolated" phenyl rings emit visible light? J. Am. Chem. Soc. 139, 16264-16272 (2017).

39. Sturala, J. et al. Excited-state aromatic interactions in the aggregation-induced emission of molecular rotors. J. Am. Chem. Soc. 139, 17882-17889 (2017).

40. Bhattacharya, A., Chatterjee, S., Prajapati, R. \& Mukherjee, T. K. Sizedependent penetration of carbon dots inside the ferritin nanocages: evidence for the quantum confinement effect in carbon dots. Phys. Chem. Chem. Phys. 17, 12833-12840 (2015).

41. Tian, Z. et al. Full-color inorganic carbon dot phosphors for white-lightemitting diodes. Adv. Opt. Mater. 5, 1700416 (2017).

42. Shimazaki, Y., Yajima, T., Takani, M. \& Yamauchi, O. Metal complexes involving indole rings: structures and effects of metal-indole interactions. Coord. Chem. Rev. 253, 479-492 (2009).

43. Dong, J. J., Shokes, J. E., Scott, R. A. \& Lynn, D. G. Modulating amyloid selfassembly and fibril morphology with $\mathrm{Zn}(\mathrm{II})$. J. Am. Chem. Soc. 128, 3540-3542 (2006).

44. Micklitsch, C. M. et al. Zinc-triggered hydrogelation of a self-assembling $\beta$-hairpin peptide. Angew. Chem. Int. Ed. 123, 1615-1617 (2011).

45. Faller, P., Hureau, C. \& Berthoumieu, O. Role of metal ions in the selfassembly of the Alzheimer's amyloid- $\beta$ peptide. Inorg. Chem. 52, 12193-12206 (2013).

46. Noy, D. et al. Zinc-amyloid $\beta$ interactions on a millisecond time-scale stabilize non-fibrillar Alzheimer-related species. J. Am. Chem. Soc. 130, 1376-1383 (2008).

47. Yang, D., Duan, P. F., Zhang, L. \& Liu, M. H. Chirality and energy transfer amplified circularly polarized luminescence in composite nanohelix. Nat. Commun. 8, 15727 (2017).

48. Tan, Y. N., Lee, J. Y. \& Wang, D. I. Uncovering the design rules for peptide synthesis of metal nanoparticles. J. Am. Chem. Soc. 132, 5677-5686 (2010).

49. Soares, D. M., Wasle, S., Weil, K. G. \& Doblhofer, K. Copper ion reduction catalyzed by chloride ions. J. Electroanal. Chem. 532, 353-358 (2002).

50. Pinotsi, D. et al. Proton transfer and structure-specific fluorescence in hydrogen bond-rich protein structures. J. Am. Chem. Soc. 138, 3046-3057 (2016).

51. Sigel, H. \& Martin, R. B. Coordinating properties of the amide bond. Stability and structure of metal ion complexes of peptides and related ligands. Chem. Rev. 82, 385-426 (1982).

52. Makam, P., Senanayak, S. P., Narayan, K. S. \& Govindaraju, T. Multi-stimuliresponsive charge-transfer hydrogel for room-temperature organic ferroelectric thin-film devices. J. Am. Chem. Soc. 138, 8259-8268 (2016).

53. Mohan, S., Puviarasan, N. \& Bakkialakshmi, S. Vibrational spectra and analysis of tryptophan. Asian J. Chem. 11, 1137 (1999).

54. Wang, X. et al. Synthesis and characterization of peptide nucleic acidplatinum nanoclusters. Nanotechnology 17, 1177 (2006).

55. Alivisatos, A. P. Semiconductor clusters, nanocrystals, and quantum dots. Science 271, 933-937 (1996).

56. Tao, K., Levin, A., Adler-Abramovich, L. \& Ehud, G. Fmoc-modified amino acids and short peptides: simple bio-inspired building blocks for the fabrication of functional materials. Chem. Soc. Rev. 45, 3935-3953 (2016).
57. Burley, S. K. \& Petsko, G. A. Aromatic-aromatic interaction: a mechanism of protein structure stabilization. Science 229, 23-29 (1985).

58. Pinotsi, D., Buell, A. K., Dobson, C. M., Schierle, G. S. K. \& Kaminski, C. F. A label-free, quantitative assay of amyloid fibril growth based on intrinsic fluorescence. ChemBioChem 14, 846-850 (2013).

59. Sytnyk, M. et al. Cellular interfaces with hydrogen-bonded organic semiconductor hierarchical nanocrystals. Nat. Commun. 8, 91 (2017).

\section{Acknowledgements}

This work was supported in part by the European Research Council under the European Union Horizon 2020 research and innovation program (no. 694426) (E.G.), HUAWEI company (E.G.), the NIH research grant (R01-CA226251) (M.Z.), the Office of Naval Research (ONR: M.Z.), the Research Supported by the CAS/SAFEA International Partnership Program for Creative Research Teams (J.L.), and the Ohio State University Comprehensive Cancer Center (Pelotonia Postdoctoral Fellowship to Z.F.). The authors thank Dr. Miri Kazes for experimental assistance, Dr. Sigal Rencus-Lazar for language editing, and the members of the Zhang, Gazit and Li laboratories for helpful discussions

\section{Author contributions}

M.Z., E.G., and J.L. conceived and designed the work; K.T. and Z.F. conducted SEM AFM, FTIR, and MS characterizations; K.T. and P.M. conducted measurements of visible fluorescence, UV-Vis absorbance and NMR; Z.F. conducted measurements of TEM and NIR fluorescence; Z.T. and S.N.Q. performed LED fabrication and characterizations; K.T. and R.A. conducted lifetime measurements; M.S.S. performed theoretical calculations; L.S., M.R., S.S.-N., D.H., Z.P., S.G., J.M., and F.Y. performed cytotoxicity test and in vivo bio-imaging experiments. K.T. and Z.F. coordinated all the work, analyzed the results, wrote, and edited the manuscript. All authors discussed and commented on the manuscript.

\section{Additional information}

Supplementary Information accompanies this paper at https://doi.org/10.1038/s41467018-05568-9.

Competing interests: The authors declare no competing interests.

Reprints and permission information is available online at http://npg.nature.com/ reprintsandpermissions/

Publisher's note: Springer Nature remains neutral with regard to jurisdictional claims in published maps and institutional affiliations.

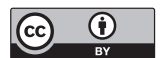

Open Access This article is licensed under a Creative Commons Attribution 4.0 International License, which permits use, sharing, adaptation, distribution and reproduction in any medium or format, as long as you give appropriate credit to the original author(s) and the source, provide a link to the Creative Commons license, and indicate if changes were made. The images or other third party material in this article are included in the article's Creative Commons license, unless indicated otherwise in a credit line to the material. If material is not included in the article's Creative Commons license and your intended use is not permitted by statutory regulation or exceeds the permitted use, you will need to obtain permission directly from the copyright holder. To view a copy of this license, visit http://creativecommons.org/ licenses/by/4.0/.

(C) The Author(s) 2018 